Cahiers de recherches médiévales

\title{
La poétique de la lettrine dans Le Roman de Silence
}

\section{Danièle James-Raoul}

\section{OpenEdition}

Journals

Édition électronique

URL : https://journals.openedition.org/crm/2322

DOI : $10.4000 / \mathrm{crm} .2322$

ISSN : 1955-2424

Éditeur

Honoré Champion

Édition imprimée

Date de publication : 30 décembre 2005

Pagination : 227-245

ISSN : $1272-9752$

Référence électronique

Danièle James-Raoul, «La poétique de la lettrine dans Le Roman de Silence », Cahiers de recherches médiévales [En ligne], 12। 2005, mis en ligne le 30 décembre 2008, consulté le 15 décembre 2022. URL : http://journals.openedition.org/crm/2322 ; DOI : https://doi.org/10.4000/crm.2322

Ce document a été généré automatiquement le 15 décembre 2022.

Tous droits réservés 


\title{
La poétique de la lettrine dans Le Roman de Silence
}

\author{
Danièle James-Raoul
}

1 Que le Roman de Silence, revendiqué par un certain Heldris de Cornuälle, nous ait été transmis dans un seul manuscrit, le manuscrit de Lord Middleton Mi.LM.6, conservé à la Bibliothèque de Nottingham, invite à s'intéresser de près aux options prises dans sa présentation textuelle, unique réalité que nous possédions de cette œuvre. Reprenant les données essentielles de la description donnée par W. H. Stevenson ${ }^{1}$ et F. A. Cowper ${ }^{2}$, Lewis Thorpe rapporte notamment la présence de grandes capitales peintes, le plus souvent alternativement, en rouge ou en bleu, chacune d'elles étant ornée, selon un usage courant, d'un filigrane de l'autre couleur que la sienne ${ }^{3}$. Toujours placées en début de vers, ces lettrines sont en général de trois unités de réglure, creusant un alinéa, quelques-unes d'entre elles s'étalant sur six à dix unités, voire plus, quand leur haste montante ou descendante se prolonge par des fioritures dans les couloirs en marge des colonnes d'écriture. Quatorze miniatures, dont onze semblent de la même main, les accompagnent, dans les folios $188 \mathrm{v}^{\circ}-223 \mathrm{r}^{\circ} \mathrm{du}$ manuscrit considéré, les remplacent même pour douze d'entre elles, et permettent, tout comme l'examen de la langue ou des sources, de dater le manuscrit de la fin du XIII ${ }^{\mathrm{e}}$ siècle, peut-être plus précisément de son troisième quart. Alors que les miniatures ont suscité un certain intérêt, et d'abord chez Lewis Thorpe comme éditeur du roman ${ }^{4}$, ces 128 lettrines, qui scandent le texte en paragraphes, ont été jusque-là presque ignorées de la critique ${ }^{5}$. Pourtant, leur distribution témoigne que le premier lecteur de ce roman a été très attentif à la poétique mise en œuvre : le copiste, qu'il ait été guidé ou non par l'auteur, nous donne en effet de précieuses indications sur la structuration de l'histoire, et, partant, sur l'importance de certains thèmes ou de certains choix, libres ou contraints, de la versification.

2 Avec les miniatures, les lettrines s'imposent comme une ponctuation rythmant la page manuscrite par les déchirures graphiques qu'elles produisent, à l'instar des alinéas dans nos textes modernes: elles sont un élément essentiel du paratexte, dont l'importance a été soulignée de longue date ${ }^{6}$. Elles indiquent des temps forts, marqués 
par une rupture narrative ou dramatique, ou, comme les didascalies dans un texte théâtral, soulignent une posture actantielle, un changement d'intonation, quand elles surviennent en liaison avec le discours. Dans son édition d'Érec et Énide de Chrétien de Troyes, Mario Roques, par exemple, a souligné naguère comment ces lettrines sont de véritables guides de lecture :

Ces initiales marquent, en général, le début d'épisodes ou de développements nouveaux, mais de façon assez irrégulière; elles sont plus nombreuses dans la première moitié (trente-neuf) que dans la seconde (vingt seulement) où l'on peut ensuite trouver des suites de 500 vers sans aucune coupure. Il apparaît dans l'ensemble que ces initiales annoncent moins les moments successifs de l'action que des épisodes mettant en relief les acteurs et leurs attitudes diverses ${ }^{7}$.

Précisant ces données, Françoise Gasparri, Geneviève Hasenohr et Christine Ruby ${ }^{8}$ ont constaté que, dans les différents manuscrits contenant Érec et Énide, les grandes majuscules s'appuient le plus souvent :

(1) sur des connecteurs, jetés en tête de vers, plus rarement situés au milieu du vers. L'ensemble des manuscrits accorde, et de loin, la préférence aux outils temporels, puis viennent les outils consécutifs et enfin les outils spatiaux. Parfois, ce sont de simples groupes nominaux circonstanciels qui sont ainsi marqués.

(2) sur la description définie d'un personnage (le héros ou un personnage qui relève du monde féodal et courtois); la lettrine met en valeur un nom propre, sujet de l'action ${ }^{9}$ ou apostrophé.

(3) sur le passage du récit au discours, plus rarement sur un changement d'interlocuteurs.

(4) en tête d'une description.

(5) de manière originale, chez Guiot, pour signaler un passage émotionnellement fort.

4 Comme le notent ces critiques, "aucun "marquage" par une lettrine n'est fait de manière systématique ${ }^{10} »$. Cependant, la combinaison de certains des éléments relevés précédemment conduit en définitive à privilégier le marquage des séquences qui débutent par un changement spatio-temporel des héros.

Dans le Bel Inconnu de Renaut de Beaujeu, conservé dans un seul manuscrit, Yan Greub corrobore la validité de ces résultats : il constate aussi une répartition inégale des 92 lettrines présentes et il enregistre, à son tour, leur diminution au fil de l'œuvre manuscrite ; il souligne que l'épisode central du roman (le Fier Baiser) n'est pas marqué par des lettrines, mais que celles-ci l'annoncent et le suivent en grand nombre; par ailleurs, l'immense majorité de ces grandes capitales est explicable par l'un des critères avancés traditionnellement, la structuration temporelle dominant ${ }^{11}$.

6 À l'évidence, les lettrines délimitent ainsi des pans textuels caractérisés par une impulsion narrative forte et par une certaine unité. Elles sont des agents de la cohésion littéraire. Les études précédemment citées offrent de solides bases de comparaison pour apprécier ce qu'offre le manuscrit de Lord Middleton conservant le Roman de Silence : l'Annexe située à la fin de cet article récapitule les données proposées.

7 Envisagée au plan global, macrostructural, du manuscrit, la distribution des lettrines apparait exemplaire de rigueur et d'intelligence du texte. Contrairement à ce que l'on observe dans plusieurs autres manuscrits, les 128 grandes capitales, que je considère toutes, indépendamment de leur taille, sont relativement bien réparties sur l'ensemble du roman, et elles sont renforcées par 14 miniatures, qui, à deux exceptions près ${ }^{12}$, les suppléent; leur nombre ne fléchit pas vers la fin, comme cela se produit souvent ${ }^{13}$. Si 
l'on considère l'ensemble lettrines-miniatures (soit: lettrines présentes-lettrines absentes), une coupure graphique devrait être enregistrée, dans l'absolu, environ tous les 47 vers, selon une fréquence relativement élevée, puisque chaque page pleine accueille deux colonnes de 48 lignes. L'écart type entre deux lettrines est, en fait, bien sûr variable, de 276 vers (v. 879-1155) à 6 vers (v. 6299-6305), avec, dans ce dernier cas, présence d'une miniature de suppléance à la première borne.

8 Le prologue est ainsi délimité, encadré par une miniature qui dispense de la lettre initiale $\mathrm{M}$ et par la grande majuscule $\mathrm{E}$ qui lui donne visuellement sa clôture, en ouvrant le récit proprement dit. L'épilogue, en revanche, où l'auteur-narrateur confisque de nouveau le discours pour prendre congé de son auditoire (v. 6684-6706), ne se distingue pas du dénouement de l'histoire (v. 6669-6683), dont il apparaît comme un prolongement textuel direct: en posant une seule lettrine $\mathrm{D}$ à l'initiale de cet ensemble final, le copiste choisit d'en sceller l'unité, instaurant une seule et même clôture à ce qui est, au plan diégétique, duel. En dépit de leur essence rhétorique similaire, essentiellement phatique, les deux seuils de l'œuvre ne sont donc pas traités sur le même mode par le copiste, le début étant pointé en clair comme un moment essentiel du texte littéraire, tandis que la fin, qui parait un lieu moins déterminé, plus fragile, offre aussi, comme tel, un plus vaste espace de liberté : on retrouve ici les données précises des études de style historique ${ }^{14}$. De même, la coupure narrative, qui organise le roman généalogique et fait succéder aux aventures des parents celles de leur enfant est ainsi soulignée, au vers 1651, alors qu'elle est déjà appuyée stylistiquement sur une métalepse (« Chi le lairons del mort ester ${ }^{15}$ »).

9 Trois pages, $193 \mathrm{r}^{\circ}, 193 \mathrm{v}^{\circ}$ et $214 \mathrm{r}^{\circ}$, sont dépourvues de cette présence habituelle des lettrines ${ }^{16}$. Deux longs passages sont ainsi dégagés visuellement comme unités narratives, dotées d'une ouverture et d'une clôture, attirant l'attention par le parti pris du copiste. Le premier (v. 879-1155) correspond précisément à la scène où Cador et Eufémie s'avouent leur amour, depuis le lapsus commis par la jeune fille jusqu'au baiser final et à la promesse réciproque de soi : c'est le dénouement de l'amour naissant, conté dans les vers précédents, avant l'obtention de sa concrétisation par le mariage. Le double choix, et de ne pas vouloir briser cet ensemble de vers, de très loin le plus long du roman, et de ne pas y intégrer le commentaire du narrateur qui rompt la diégèse dans les vers suivants immédiats (v. 1155-1198), met en valeur une scène privilégiée dans les romans médiévaux et attendue comme un morceau de bravoure par le public, depuis l'Énéas notamment; or, ce roman antique est l'une des sources d'inspiration de l'auteur ${ }^{17}$ et peut-être a-t-on là une indication supplémentaire de la dette conçue. Pour être moins clos sur lui-même, le second passage (v. 4919-5069, à moins qu'il ne faille considérer les vers 4919-5114) comporte, lui aussi, une unité narrative et thématique certaine, correspondant à un rebondissement de l'histoire : alors que le roi Ébain vient d'apprendre que sa lettre au roi de France a été falsifiée, portant l'arrêt de mort de Silence, deux responsables successifs de la missive sont envisagés. Le premier, immédiatement convoqué et puni à tort, comme le sait l'auditeur-lecteur, est le chancelier; celui-ci finit par comprendre que seule la reine peut être la véritable responsable de ce qui lui est reproché et qu'elle a sans doute procédé à la substitution de sa lettre, cherchant à se venger de Silence pour une raison qu'il ignore. Avec l'apparition de la miniature au vers 5069, le passage s'interrompt au moment où le roi, que le chancelier a demandé à voir, somme celui-ci de parler; si l'on ne tient pas compte de cette miniature qui, cependant, arrête la lecture comme le ferait une lettrine, la lecture est conduite jusqu'à la lettrine du vers 5114, qui apporte la 
résolution de cette péripétie narrative. A posteriori cependant, l'issue adoptée dans ce paragraphe n'apparaît que partielle et est remise en question, parce qu'Ébain préfère garder secrète la mise au jour du subterfuge, sans que la reine Eufeme soit inquiétée et châtiée: l'histoire peut ainsi continuer... Dans la première option, l'unité est globalement moindre, mais elle a le mérite d'attiser le suspens et de mettre le lecteur sur le grill en attente de réponse; dans la seconde option, elle donne au passage une clôture pour lors apparemment parfaite. On voit aussi par là que, loin de se concurrencer, lettrines et miniatures vont dans le même sens en tant que respiration du texte, et que le copiste emploie celles-ci pour épauler celles-là.

Inversement, dans un peu plus du quart des pages, l'œil est non moins surpris par une abondance remarquable des lettrines et, éventuellement, d'une miniature: quatre lettrines dans les folios $204 \mathrm{v}^{\circ}, 205 \mathrm{v}^{\circ}, 216 \mathrm{r}^{\circ}, 221 \mathrm{v}^{\circ}$; trois lettrines et une miniature dans le folio $206 \mathrm{v}^{\circ}$; trois lettrines dans les folios $188 \mathrm{v}^{\circ}, 201 \mathrm{v}^{\circ}, 203 \mathrm{v}^{\circ}, 204 \mathrm{r}^{\circ}, 206 \mathrm{r}^{\circ}$, $207 \mathrm{v}^{\circ}, 208 \mathrm{v}^{\circ}, 216 \mathrm{v}^{\circ}, 218 \mathrm{r}^{\circ}, 222 \mathrm{r}^{\circ}$; deux lettrines et une miniature dans les folios $195 \mathrm{v}^{\circ}, 203 \mathrm{r}^{\circ}, 211 \mathrm{r}^{\circ}, 221 \mathrm{r}^{\circ}, 222 \mathrm{v}^{\circ}$. Plus que la distribution dans le détail de ces marques graphiques qui attirent indubitablement l'attention, c'est la concentration du phénomène sur deux, trois ou quatre pages adjacentes qui est frappante, suggérant leur parenté textuelle. Par cette présence, les vers 2873-3233 sq. ( $\left.f^{\circ s} 203 \mathrm{r}^{\circ}-204 \mathrm{v}^{\circ}\right), 3339-3611$ sq. $\left(f^{\circ \circ} 205 \mathrm{v}^{\circ}-206 \mathrm{v}^{\circ}\right), 5327-5485$ sq. $\left(\mathrm{f}^{\circ} \mathrm{s} 216 \mathrm{r}^{\circ}-216 \mathrm{v}^{\circ}\right), 6299-6651$ sq. $\left(\mathrm{f}^{\circ \circ} 221 \mathrm{r}^{\circ}-222 \mathrm{v}^{\circ}\right)$ sont ainsi marqués comme de grands ensembles narratifs. De fait, l'histoire connaît, dans ces pages, de brusques accélérations. La richesse en péripéties, qui caractérise ces passages est, précisément, traduite au plan visuel par la multiplication des lettrines, secondées par les miniatures. Chacun des ensembles narratifs ainsi dégagé relance l'aventure et l'action: mise à exécution par Silence de son projet de partir avec les jongleurs, la jeune fille travestie devenant, irrésistiblement, un excellent ménestrel, célèbre et apprécié (v. 2873-3232 sq.), ce qui va entraîner la jalousie haineuse de ses compagnons; projet meurtrier des jongleurs éventé par Silence qui, avant tout préjudice, décide de retourner dans son pays, où elle est reconnue par un vieillard (v. 3339-3611 sq.), avant de l'être par son père et de gagner la cour du roi ; début du combat sanglant des Anglais contre la rébellion des comtes (v. 5327-5485 sq.), où la victoire, remportée grâce à Silence, va entrâner son retour à la cour et la nouvelle tentative de séduction de la reine; révélations de Merlin (v. 6299-6651 sq.), qui apportent le dénouement. Parce que l'abondance graphique relevée sur plusieurs pages successives n'a, cependant, rien de systématique dans l'ensemble de l'œuvre et que, $a$ contrario, de longs épisodes, comparables par leur contenu et leur valeur dramatique, ne sont pas étiquetés de la sorte, le libre-arbitre du copiste peut ici sembler patent. Mais cette impression doit être amplement modérée, parce que, de manière quasi exhaustive, tous les moments clés du roman prennent place dans des folios marqués, isolément, par trois lettrines ou deux lettrines et une miniature : le début de l'œuvre $\left(f^{\circ} 188 v^{\circ}\right)$; la décision du mariage entre Cador et Eufémie $\left(f^{\circ} 195 \mathrm{v}^{\circ}\right)$; la fin du débat entre Nature et Noreture et le triomphe de Raison chez l'adolescente $\left(f^{\circ} 201 \mathrm{v}^{\circ}\right)$; la première tentative de séduction de Silence par la reine Eufeme $\left(f^{\circ} 207 v^{\circ}\right)$, puis la deuxième $\left(f^{\circ} 208 \mathrm{v}^{\circ}\right)$; l'arrivée de Silence à la cour de France, avec la découverte du contenu de la lettre après le baiser de paix $\left(f^{\circ} 211 r^{\circ}\right)$; la troisième tentative de séduction de la reine qui, calomniant Silence, demande au roi de se débarrasser définitivement de cet individu en l'envoyant à la recherche de Merlin ( $\left.f^{\circ} 218 r^{\circ}\right)$.

11 Pour finir, un dernier élément concernant la distribution de cette ponctuation graphique et ornementale peut être noté ou rappelé18. Un peu moins de la moitié des 
pages manuscrites affichent deux lettrines, ou une lettrine et une illustration ou encore, avec ostentation, des lettrines géminées. Ce choix, me semble-t-il, visualise de manière étonnante le motif de la gémellité, du double ou de la dualité, omniprésent et fondateur dans l'histoire racontée ${ }^{19}$. Sans doute n'est-il pas anodin de constater de surcroît que, au sein des capitales géminées, c'est la lettre L qui est inscrite avec insistance : différente de toutes les autres lettres parce qu'elle se loge dans un alinéa épais d'un vers ou deux seulement, exceptionnelle par sa taille qui dépasse fréquemment la dizaine d'unités de réglure et que les fioritures accrochées à la haste prolongent encore, cette lettre est 8 fois géminée. Tandis que le $\mathrm{S}$ apparaît 19 fois, le $\mathrm{A}$ 13 , le $\mathrm{O} 10$ et le D 8, elle est la lettre qui compte - et de loin - le plus grand nombre d'occurrences ${ }^{20}$, avec un total de 41 ; elle ne cède jamais sa place à une miniature. Comme pour donner la tonalité majeure dans laquelle la composition du roman est à lire, elle est l'emblème de cette vie qui, assurément pour l'homme médiéval, anime les lettres $^{21}$. Ainsi, l'écriture ornementale semble porter un regard critique, voire ironique, sur la fiction. Silence a beau passer aux yeux de tous pour un garçon, le livre affirme avec supériorité qu'il n'est pas dupe des apparences, et il ne cesse d'exhiber en grand la féminité cachée par la fiction : «il », dit le texte ; lisez « elle », L, dit le livre ${ }^{22}$ !

Dans le détail, au plan microstructural, la distribution des lettres n'est pas moins éloquente par l'originalité qu'elle affiche ${ }^{23}$. Comme dans de nombreux manuscrits, elle marque en pointillés une structuration qui prend appui sur des éléments essentiels de la narration, mais d'une façon qui lui est personnelle. La présence des connecteurs n'est pas ici primordiale, comme si le copiste voulait suggérer que les structures de la fiction reposaient de préférence sur d'autres emplois: le plan temporel est étonnamment délaissé, alors que la chronologie gouverne nettement le déroulement de la diégèse ; le plan spatial apparaît une seule fois ${ }^{24}$; les catégories logiques sont quasiment ignorées et deux connexions consécutives apparaissent seulement ${ }^{25}$. Le plan actantiel et le plan discursif dominent dans ce manuscrit, de manière écrasante. Quant au plan métaleptique, enfin, même s'il n'est pas aussi favorisé que les deux précédents, il bénéficie, non sans nouveauté, des faveurs du copiste.

13 La structuration temporelle, traditionnellement fort appuyée par les lettrines dans les copies manuscrites, est ici largement sacrifiée, avec 27 cas seulement (indiqués par T dans le tableau figurant en Annexe). Sans nouveauté, elle repose sur la mise en avant d'un connecteur adverbial (Atant, Encor, $\mathrm{Or}^{26}$ ), conjonctif (Ançois que, Quant), d'un groupe nominal complément circonstanciel, prépositionnel (Droit al tierc jor, Contre le soir, D'illuec al tierc jor) ou absolu (Un jor), ou encore d'une mention chronologique (Li jors apert). Le plus souvent, ces indications premières sont suivies dans le vers de la présence d'un actant et un mouvement y est enregistré, ou une nouveauté, comme le montrent les quelques exemples suivants :

Atant i sorvint Noreture (v. 2547)

Ainçois que l'aube soit veüe, (v. 839)

Contre le soir li rois repaire : (v. 5747)

Un jor repairent de Gascoigne, (v. 3215)

Li jors apert et Eufemie (v. 879)

14 La structuration actantielle, marquée par les lettrines ou les miniatures dans 77 cas (notés A dans le tableau final), affirme sa prééminence avec plus de force que ne le propose la tendance courante : l'ornement graphique intervient porté par un nom de personnage, toujours sujet de l'action ou apostrophé, dirigeant la parole adressée. Il s'agit d'un nom propre de personne (Ebans, Cador, Silences, Adans, Merlins), d'une entité 
allégorique (Amors, Raisons), d'un titre (Maistres), d'un pronom (Il, Li altres, Uns, Cil), d'une description identifiante commençant alors par un caractérisant ou un déterminant, sauf s'il s'agit d'une apostrophe (Li rois, La roïne, Les .xxx., Li cuens de..., Bials sire Dex, Segnor, Bials sire ciers, Tolte la cors, Li senescals, Li jogleör, Amis, Uns viellars, La/ Ceste dame, Sire, Li François, Li .iiii. conte desloial) :

Ebans fu ja rois d'Engletiere, (v. 107)

Amors m'a mis en noncaloir, (v. 787)

Cil voit celui, si l'enorta (v. 5913)

La dame son col desafice (v. 3791)

Segnor, jo voel que Cador ait (v. 1275)

Pour plus de la moitié d'entre elles, ces différentes mentions s'organisent autour du trio actantiel principal : 16 occurrences pour le roi Ébain, toujours convoqué par son titre ou appelé comme souverain, sauf lors de sa première mention; 14 occurrences pour Silence, exclusivement convoquée sous son nom propre; 8 occurrences pour la reine Eufeme, toujours désignée par une désignation non rigide, qui l'inscrit dans le jeu anaphorique comme reine ou comme dame. Cador et Eufémie arrivent loin derrière, ne totalisant à eux deux que 5 mentions: c'est confirmer que, dans ce roman généalogique, les amoureux de la première partie ne sont là que pour donner naissance à celle dont la seconde partie contera les aventures.

La structuration discursive, avec 66 cas, est quantitativement soulignée par un nombre très élevé de lettrines, par rapport à ce que l'on observe ailleurs : l'introduction du discours, le changement d'interlocuteurs, la reprise du récit avec l'abandon du discours sont ainsi mis en valeur. Cette armature peut éventuellement être associée à la structure temporelle ou actantielle: la cohérence textuelle en est renforcée. Caractérisés par des marquages spécifiques, les embrayeurs ou les déictiques, et par toute la palette possible des différentes modalités, le discours rapporté directement, auquel il faut adjoindre le discours indirect libre, perturbe fondamentalement la narration en l'animant. Lettrines et miniatures le soulignent à plaisir : la parole est bien au cœur du Roman de Silence ${ }^{27}$.

17 C'est le début ou l'introduction du discours direct qui est ainsi marqué : on en compte 21 occurrences (notées dD dans le tableau final figurant en Annexe). La lettrine (ou la miniature) indique visuellement, en avant-poste, ce que le plan stylistico-syntaxique signifie toujours autrement, par un verbe déclaratif de présentation, lourdement mis en avant plus que situé en incise, ou bien par une adresse, sauf exception ${ }^{28}$ :

«Bials sire Dex, ki formas home, (v. 427)

Li rois a dit, voiant trestols : (v. 1499)

Cho dist Nature : « Jo m'en duel» (v. 1921)

Cho dist li rois : «Ma douce suer, » (v. 4281)

Le changement d'interlocuteurs, avec 11 occurrences seulement, est rarement marqué d'une lettrine. Dans 7 cas (étiquetés $\mathrm{fD}-\mathrm{dD}$ dans le tableau), le passage est laborieusement effectué à l'aide d'une sorte de collage qui fait se succéder deux discours formellement dissociés, dont le second est introduit par du récit: la lettrine signale le relais de l'un à l'autre. Elle intervient le plus souvent en tête du vers où reprend formellement le récit, le discours ayant été congédié dans le vers précédent, mais la suite du vers est tournée vers un nouveau discours, grâce à la présence systématique d'un verbe déclaratif :

«Dunt dist la contesse et la dame : « (v. 2083)

«Silences respont : « Tort avez, (v. 6265) 
Quand les répliques s'enchaînent immédiatement, le discours transmis en acquiert vivacité et naturel : l'effet produit est sensible, surtout par comparaison avec l'option précédente ; pourtant, on n'en relève que 4 cas (notés $\mathrm{CD}$ dans le tableau final). Le début $\mathrm{du}$ vers est doublement signalé comme nouvelle réplique, stylistiquement par une adresse à celui qui vient de parler et graphiquement par la lettrine ou la miniature ; éventuellement, la suite du vers renforce encore ce marquage par la présence d'un verbe déclaratif en incise. Trois cas sont simples, tandis que le quatrième (v. 3501) est plus délicat ; j'y reviendrai plus loin :

- Bials sire ciers, cho dist la dame, (v. 1695)

- Amis, font il, ne vos cremés. (v. 3437)

- [S]ire, ma dame vint a moi. (v. 5069)

48 lettrines correspondent à un retour au récit, le discours direct s'étant terminé dans le vers précédent: en l'absence commune de marque lexicale ou syntaxique signifiant le changement, le discours direct se terminant souvent abruptement, la lettrine est ici plus qu'ailleurs un signal commode et économique pour alerter le récepteur. Que le copiste ait préféré privilégier par les lettrines la fin du discours plutôt que son début témoigne de son esprit pratique et logique: dans le champ discursif, les lettrines possèdent donc sous sa plume une orientation rétroverse plutôt que proverse. Les exemples abondent, mais l'un d'eux retient particulièrement l'attention, où l'abandon du discours indirect libre est souligné, avec subtilité :

Trestolt li frons lor en degotte

C'on por un garçon les debotte.

Silences estoit si bials

N'ert pas garçons, mais damoisials. (v. 3161-3164)

21 La lettrine que le copiste choisit de placer en retournant au récit est, au plan graphique, parfaitement à l'unisson de la rupture narrative à l'œuvre et elle alimente une esthétique du contrepoint: passage paratactique du discours rapporté indirectement au récit, avec modification de l'énonciateur des propos; changement de plan presque métonymique, qui fait passer des jongleurs vitupérant contre Silence à celui-ci; changement thématique, qui rectifie l'injure par un compliment appuyé ; reprise de la qualification ignominieuse injustifiée et, comme telle, déniée dans une structure formellement oppositive, en réalité contradictoire.

Enfin, 25 lettrines (présentes ou éclipsées par une miniature) appuient la structuration métaleptique, avec une récurrence confirmant la place importante que se donne l'auteur-narrateur dans ce roman. 11 d'entre elles (notées $\mathrm{dM}$ dans le tableau situé en Annexe) marquent l'interruption de la diégèse et donnent le passage à une métalepse narrative, tandis que 14 opèrent le retour à la diégèse, le discours métaleptique ayant pris fin dans le vers précédent ${ }^{29}$ (elles sont notées $\mathrm{fM}$ dans le tableau final). Dans l'un et l'autre cas, indépendamment du fait que la lettrine marque une rupture liée au vers qui la porte (valeur proverse) ou au vers qui la précède (valeur rétroverse), l'instance narrative est explicite ${ }^{30}$ et fait intervenir la première personne du singulier. En surplomb de l'histoire, l'auteur-narrateur peut manifester un point de vue omniscient et asserter la vérité de ce qu'il dit, parce qu'il connaît «l'histoire qui retrace la vie de Silence ${ }^{31} »$ (v. 2657-2658). Il en assume la régie : il veille à ne dire que ce qui est essentiel (v. 3539) ou possible (v. 3654), il contribue aux transitions en annonçant la suite (v. 733, $1576,1795,2987,3710,3742)$ ou en mettant fin à un épisode (v. 1651, 1861, 2256). Il anime aussi, comme un bateleur, la narration, apostrophant son public à la manière de ce qui se pratique dans le genre épique, sollicitant son écoute attentive ou 
rafraîchissant sa mémoire à propos d'un épisode (v. 2689, 3095, 3127, 4036, 5327, 5878). $\mathrm{Au}$ cœur de l'histoire, il commente l'événement (v. 2400), se pose en moraliste, analyste rompu au fonctionnement des sentiments (v. 1432, 3924), en écrivain particulièrement consciencieux (v. 2358) ou modeste (v. 5296), ou encore en témoin (v. 4498). J'en cite quelques exemples:

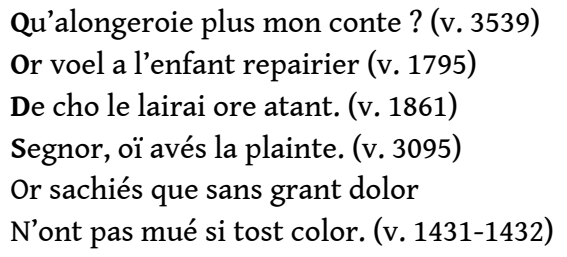

Ce bel ensemble, ainsi structuré par les lettrines, laisse une petite place à quelques cas plus résiduels. Une douzaine d'entre eux sont relativement banals. Les vers pointés par ces lettrines ont une teneur globalement dramatique ou descriptive, ils introduisent une nouvelle phase de l'action ou une scène particulière. Sont ainsi notés la relance d'un discours déjà commencé, avec introduction d'un nouveau mouvement de l'argumentation logique (v. 4781), ou, de façon plus banale, un changement de plan (v. 2127, 3369, 5191, 5369). C'est enfin le descriptif, plutôt que la description ${ }^{32}$, qui est ainsi signalé. Les scènes ou les propos ont, le plus souvent, un caractère d'une part plaisant, par leur naïveté (v. 645) ou l'insertion d'une métaphore déplacée (v. 3009), d'autre part, intensif, voire exagéré (v. 139, 2203, 3049, 3655, 5145, 5437) :

Moult ot prodome en roi Ebain (v. 139)

Noviele atrote et si acort (v. 3009)

Sa harpe a cil bien atenpree (v. 3743)

Longe est et griés lor pasmisons (v. 3049)

Presque toutes les occurrences considérées se caractérisent par le même patron syntaxique qui antépose, en tête de phrase et de vers, le prédicat de la phrase (complément, adjectif qualificatif, participe passé) ou un adverbe intensif (Moult, Moult par). Pourtant, cet ordre des constituants de la phrase est en nette régression à partir $\mathrm{du}$ XIII ${ }^{\mathrm{e}}$ siècle et son emploi, quand il subsiste, en acquiert une valeur stylistique ${ }^{33}$; la lettrine ne fait que conforter la connotation de la syntaxe.

Plus intéressants apparaissent les trois cas restants où le copiste témoigne de surcroît, par la pose de sa lettrine, qu'il est très attentif à la versification. De façon extrêmement conservatrice, jamais une lettrine ne rompt un couplet de vers liés par la rime. Pourtant, si l'usage de briser le couplet rimique par une lettrine est ignoré des premiers manuscrits, il commence à se répandre, au cours du XIII ${ }^{e}$ siècle ${ }^{34}$. Ainsi, alors que le manuscrit de Guiot n'en offre pas d'exemples dans son Conte $d u$ Graal, le manuscrit correspondant de Berne 354 en offre une occurrence (v. 1480), le manuscrit BNF fr. 12786 du Roman de la Rose de Guillaume de Lorris en propose deux (v. 156, 1923), et le manuscrit de Londres, Brit. Mus. Add. 36614 de la Première Continuation de Perceval en propose de très nombreuses occurrences (v. 458, 1158, 1520, 1712, 1984, 2088, 2240, 2352, etc.). En tant que versificateur, Heldris de Cornuälle pratique, de fait, cet usage de la brisure du couplet, apparu dès le début du XII ${ }^{e}$ siècle et courant depuis l'époque du maître champenois; son copiste, lui, s'y refuse avec obstination ${ }^{35}$. De ce fait, trois lettrines $(3501,4719,5835)$ semblent comme posées en retard d'un vers, de manière décalée : leur justification ne se trouve pas dans le vers porteur et la rupture attendue prend place au début du vers précédent. La lettrine possède ici une valeur rétroverse accusée, qui incite à se référer au vers pénultième : or, c'est toujours ce vers qui lance la 
première rime en attente de la seconde. C'est la raison pour laquelle, dans le tableau final, je fais figurer à trois reprises des indications structurelles placées entre crochets. Systématiquement, c'est la parole qui est touchée par cette discordance entre graphie et versification : seuls sont concernés les plans discursif et métaleptique. Ainsi, le vers 3501 porte une lettrine, alors que c'est le vers 3500 qui enregistre le changement d'interlocuteur ${ }^{36}$; mais parce que ce vers est lié par la rime au précédent d'une façon qui semble indéfectible, la lettrine ne l'affecte pas de sa rupture :

«Amis, fait il, cis diols est vostres.

- Il est ambeure et miens et vostres.

Or entendés a ma raison, (v. 3499-3501)

Les vers 4719 et 5835 offrent des cas tout à fait similaires, en dépit de leurs conditions discursives différentes (respectivement introduction d'un discours; fin et début d'un discours). Qu'il faille y voir une marque de rigueur, un penchant pour la tradition, un souvenir d'école ou encore une oreille particulièrement sensible à l'harmonique des couplets d'octosyllabes - le fait est là, qui mérite d'être souligné.

Si cette volonté de ne pas briser les couplets d'octosyllabes par les lettrines ou les miniatures s'avère notable dans le manuscrit du Roman de Silence, le choix de la place des lettrines, dans leur succession et en liaison avec les lettrines adjacentes, appelle encore, pour finir, quelques remarques qui confirment la personnalité d'un copiste connaisseur des traditions littéraires en matière de versification. Fondamentalement, l'enchaînement des rimes plates conduit à un déroulement en couplets d'octosyllabes qui unifient la matière, bribe par bribe, à une micro-échelle. Or, si le principe de la partition épique, en séquences assonancées, particulièrement contraignantes, a été abandonné dans l'écriture du roman en vers, depuis le XII ${ }^{e}$ siècle, des traces évidentes en subsistent, d'une part dans l'enchaînement des vers, ce que je n'examinerai que sommairement ici, et, d'autre part, dans l'enchainement des différents blocs de vers déterminés par les marques graphiques, lettrines et miniatures. L'esthétique de cet enchaînement, fondée sur différents types de reprises, s'inspire directement de l'esthétique épique et ne fait que conforter les diverses structurations précédemment observées, empêchant définitivement toute plate linéarité.

Tout d'abord, le retour d'une même note initiale et finale au sein d'un seul paragraphe, peut tenir comme dans un étau le passage, lui conférant une solide unité. C'est le principe qui guide l'écriture des laisses dont le vers d'intonation est repris par le vers de conclusion. Dans le Roman de Silence, la reprise, d'une part, ne concerne jamais l'intégralité du vers : elle porte surtout sur une lexie (le plus souvent un actant) et elle est lexicale ou thématique (sur le mode de la similitude, de l'analogie ou de l'opposition) ; l'anaphore associative est ici essentielle. D'autre part, elle intervient de manière plus lâche que dans l'épopée, parfois située aux bornes précises du paragraphe, mais parfois seulement dans les premiers et les derniers vers. Entre le cinquième et le quart des paragraphes sont ainsi bâtis :

Li rois Ebayns se part de Cestre (v. 337)

Et li rois Ebayns plaint et plore. (v. 372)

Amors m'a mis en noncaloir, (v. 787)

Com Amors le castie et blece. (v. 838)

- Amis, font il, ne vos cremés. (v. 3437)

Et cil s'en vont hastivement. (v. 3476)

Quant Noreture cho oï (v. 2587)

S'il aperçoit vostre nature. » (v. 2624) 
Dans de rares cas, non sans malice, l'identité des reprises cache l'altérité, en s'appliquant à d'autres circonstances, d'autres actants, d'autres actions. C'est ce qui se passe au vers 5878, par exemple, où l'auteur-narrateur reprend à son compte, en refrain, le propos inaugural que tenait le roi Ebain à Silence :

Or escotés que vos dirai (v. 5835)

Or escoltés confaitement : (v. 5878)

Ensuite, on observe, dès les premières séquences textuelles, un enchaînement qui rappelle l'enchaînement simple des laisses : la reprise, en tête d'un paragraphe, porte sur un élément qui se trouvait en fin du paragraphe précédent. La reprise exacte du même vers est rare : là encore, c'est plutôt une lexie qui est concernée, voire plusieurs. Le procédé de la dérivation ou du polyptote intervient ici fréquemment dans le jeu anaphorique. Parfois, la reprise se fait du dernier vers du paragraphe au premier vers du paragraphe suivant, le rebondissement du mot n'en ressortant que mieux :

Mais en prodome n'a que dire. (v. 138)

Moult ot prodome en roi Ebain (v. 139)

Parfois, l'enchaînement est, comme l'appelle Dominique Boutet en se référant aux chansons de geste, "différé37 ", les derniers vers d'un paragraphe et les premiers vers du paragraphe suivant étant concernés; le phénomène de reprise peut alors aisément gagner en ampleur et toucher plusieurs mots, tissant étroitement la transition :

Par vostre consel li donrai

Ma fille ; et si l'en somonrai,

Por acorde et por aliänce,

Qe la pais soië a fiänce. » (v. 169-72)

Rois Beghes fait Ebain savoir

S'il violt qu'il puet sa fille avoir

Por acordance de la guerre,

Et qu'il ait mis en pais la terre. (v. 173-176)

Quand la répétition intervient en tête de paragraphes successifs, les lettrines semblent alors placer comme en série les épisodes qui y sont contenus. La reprise, surtout lexicale et exacte, placée dans l'incipit, ou dans les vers immédiatement suivants, est particulièrement voyante :

Li rois Ebayns se part de Cestre (v. 337)

Li .xxx. sunt el bos estraint,

Et li rois a son duel estraint. (v. 373-374)

Del serpent moult grant joie funt.

La tieste o auls porté en ont.

Li rois a puis tant esploitié (v. 533-535)

Li rois se colce quist lassés (v. 575)

Li cuens de Cestre a lui s'atrait (v. 1309)

Li cuens de Cestre est moult voiseus : (v. 1399)

Li cuens i vint. Dist : « Dex vus salt ! (v. 1433)

[Miniature] Il oënt que li cuens de Cestre (v. 1463)

Silences dant Merlins enmainne (v. 6161)

Or a Merlins moult mal tissu. (v. 6181)

Li rois par maltalent respont :

«S'il orendroit ne le despont (v. 6225-6226)

Silences respont : « Tort avez,

Dame roïne, et ne savez

Que li rois le fist amener (v. 6265-6267)

Merlins est menés en la place. (v. 6299)

Or voit bien Merlins qu'il mourra (v. 6305) 
«Merlin, Merlin, li rois a dit (v. 6341)

Que que la dame die ou face,

Merlins n'a soig de sa manace. (v. 6377-6378)

regard deux paragraphes semblables ou proches par leur début et leur fin. À la différence des laisses similaires que le procédé évoque, le contenu de chaque épisode est cependant très variable. Par l'effet de refrain créé et le rythme ainsi insufflé, le procédé est particulièrement adéquat pour mimer une stase de l'action, comme dans le cas d'une description ou d'une situation qui a du mal à progresser. La scène qui préside à l'aveu d'amour entre Cador et Eufémie en offre un bel exemple et la fin du second paragraphe affirme clairement quelle option a été choisie dans l'alternative énoncée à la fin du paragraphe précédent :

Ançois que l'aube soit veüe,

S'en est la mescine meüe. [...]

$\mathrm{U}$ cascuns d'als son per garra,

U la mecine n'i parra. (v. 839-878)

Li jors apert et Eufemie [...]

Qu'ai[n]si sui jo l[a] vostre amie

Et qu'el mont fors [vos] nen a mie

Qui ma dolor puist estancier,

Ma santé rendre, n'avancier. » (v. 879-1154)

S'intéresser aux lettrines dans le Roman de Silence permet de mettre en lumière à quel point la cohérence et la cohésion sont particulièrement soignées dans sa mise en texte manuscrite. Sans que l'on puisse savoir ou dire si le copiste a œuvré seul ou s'il a bénéficié de l'aide de l'écrivain, et même s'il n'y a aucune systématisation dans les options prises, il opère à l'évidence d'une main d'expert, très sûre, dans sa généreuse distribution de ces marques graphiques, témoignant d'une connaissance parfaite et d'une intelligence de toute l'œuvre. Point de mire de la page où elles s'étalent, attirant l'œil, ses lettrines, secondées par les miniatures, rythment non seulement la lecture, mais, par leur présence ou leur absence, elles alertent le récepteur, en lui suggérant quels sont les temps forts de l'action, elles l'aident à se repérer, en le guidant dans sa progression de l'histoire, elles soulagent sa mémoire, enfin, en y inscrivant facilement certains vers, certains moments, ainsi marqués de leur poinçon. Plus encore, elles affichent précisément, dans ce manuscrit, la volonté qu'a le copiste, en surplomb de l'écrivain, d'exercer son art dans le respect d'une tradition métrique et littéraire léguée par l'ancien temps, et avec le projet évident de créer une véritable poétique. 


\section{Distribution des lettrines dans le Roman de Silence}

\section{ANNEXES}

Les marqueurs mentionnés sont donnés par le premier mot porteur de la lettrine initiale, inscrite ou absente ; ce qui figure dans la suite du vers n'est ici pas pris en compte. Seuls les principaux marqueurs ont été retenus dans le tableau : marqueur actantiel (abrégé en A) ; marqueur temporel (T) ; marqueur discursif (D), qu'il s'agisse de l'introduction d'un discours direct (dD), d'un changement d'interlocuteur (cD), ou du retour au récit, le discours direct s'étant terminé dans le vers précédent ${ }^{38}(\mathrm{fD})$; marqueur métaleptique $(\mathrm{M})$, avec début de la métalepse $(\mathrm{dM})$ ou retour à la diégèse, la fin de la métalepse étant enregistrée dans le vers précédent (fM). La mise entre crochets indiquent les ajustements, commentés dans l'article, qui déplacent d'un vers la lettrine et l'absence de catégorie correspond à quelques rares autres cas, également commentés.

\section{NOTES}

1. W. H. Stevenson, Report on the Manuscripts of Lord Middleton preserved at Wollaton Hall, Nottinghamshire, London, Historical Manuscripts Commission, 1911, p. 221-234.

2. F. A. G. Cowper, « Origins and peregrinations of the Laval-Middleton Manuscript », Notthingham Mediaeval Studies, 3, 1959, p. 3-18.

3. Voir Le Roman de Silence : A thirteenth-century Arthurian verse-romance by Heldris de Cornuälle, éd. L. Thorpe, 1972, p. 6. Pour des références détaillées, voir la bibliographie établie conjointement avec S. Menegaldo.

4. Ibid., p. 6-8. Voir aussi M. Bolduc, dans «Silence's Beast » et dans «Images of Romance : The Miniatures of Le Roman de Silence », Arthuriana, 12, 1, 2002, p. 101-112.

5. Elles ne sont pas toutes enregistrées ou n'apparaissent pas toutes lisiblement dans l'édition proposée par S. Roche-Madhi, notamment à cause de contraintes matérielles qui empêchent de les repérer. Du fait que chaque paragraphe est séparé du suivant par un simple blanc typographique, il suffit que l'un se termine en bas de page et que l'autre commence en haut de page pour que la séparation n'apparaisse pas et que les vers semblent s'enchaîner sans pause, indépendamment du choix manuscrit : c'est ce qui se passe pour une dizaine de paragraphes.

6. Voir notamment J.-P. Bordier, Fr. Maquère, M. Martin, «Disposition de la lettrine et interprétation des œuvres: l'exemple de la Chastelaine de Vergi», Le Moyen Âge, 79, 1973, p. 231-250 ; H. R. Runte, «Initial Readers of Chrétien de Troyes », Continuations : Essays on Medieval French Literature and Language in Honor of John Lambert Grigsby, éd. N. J. Lacy et Gl. Torrini-Roblin, Birmingham (Alabama), 1989, p. 121-131; Fr. Gasparri, G. Hasenohr et Chr. Ruby, « De l'écriture à la lecture : réflexion sur les manuscrits d'Érec et Énide», Les Manuscrits de Chrétien de Troyes, Amsterdam/Atlanta, Rodopi, 1993, tome un, p. 97-148; R. Middleton, «Coloured Capitals in the Manuscripts of Erec et Enide ", Les Manuscrits de Chrétien de Troyes, op. cit., tome un, p. 149-193; L. Evdokimova, «Disposition des lettrines dans les manuscrits du Bestiaire d'Amour ", Le Moyen 
Âge, 102, 1997, p. 465-478 et 103, 1998, p. 83-115 ; K. Busby, Codex and Context, Amsterdam/Atlanta, Rodopi, 2000, en particulier tome un, p. 182-198; Chr. Ruby, Mise en page et mise en texte dans les manuscrits des romans de Chrétien de Troyes (XIII ${ }^{e}-X^{e} V^{e}$ siècles), Thèse de Doctorat, Université de Paris-Sorbonne (Paris-IV), dir. G. Hasenohr et M. Zink, 2000.

7. M. Roques, Les Romans de Chrétien de Troyes édités d'après la copie de Guiot (Bibl. nat., fr. 794), I. Érec et Énide, Paris, Champion, CFMA, 1978, p. XLII.

8. F. Gasparri, G. Hasenohr et Chr. Ruby, « De l'écriture à la lecture : réflexion sur les manuscrits d'Erec et Enide ", art. cit., p. 97-148.

9. C'est le plus souvent un mouvement indiquant un changement de scène ou de décor.

10. F. Gasparri, G. Hasenohr et Chr. Ruby, « De l'écriture à la lecture : réflexion sur les manuscrits d'Erec et Enide ", art. cit., p. 102.

11. Y. Greub, «La structuration du Bel Inconnu vue dans son manuscrit unique », communication au XXI ${ }^{\mathrm{e}}$ Congrès International Arthurien, Utrecht, 24-31 juillet 2005.

12. Aux vers $1463\left(f^{\circ} 195 \mathrm{v}^{\circ}\right)$ et $4459\left(f^{\circ} 211 \mathrm{r}^{\circ}\right)$, la miniature ne remplace pas, comme cela se produit partout ailleurs, la première lettre de la phrase : l'œil enregistre la coupure graphique, l'analyse comme une rupture textuelle, mais le texte est intégral. On pourrait également reconsidérer la forme organisant le mouvement de chaque miniature en liaison avec la forme de la lettre qu'elle supplée précisément: on peut trouver des ressemblances parfois saisissantes et deviner, dans la miniature, la lettre disparue à l'initiale !

13. On sait que cette tendance subsistera ultérieurement dans les romans en prose.

14. Voir D. James-Raoul, «Chapitre II. Du prologue à l'épilogue : l'écriture des seuils », dans Chrétien de Troyes, la griffe d'un style, Paris, Champion, NBMA (à paraître).

15. Les corrections souhaitées par F. Lecoy dans son compte rendu de l'édition de L. Thorpe («Corrections. Le Roman de Silence d'Heldris de Cornualle», 1978) ne modifient pas, sur les vers cités dans cette étude, les leçons données par L. Thorpe et confirmées ultérieurement par S. Roche-Madhi.

16. Visuellement, sur chaque page, sauf sur la page initiale, chaque miniature est toujours accompagnée d'une, deux ou trois lettrines.

17. Voir, par exemple, S. Roche-Madhi dans l'introduction donnée à son édition (p. XII-XIV) et L. K. Stock, "Arms and the (Wo)man' in Medieval Romance: The Gendered Arming of Female Warriors in the Roman d'Eneas and Heldris's Roman de Silence », 1995.

18. Je m'y suis intéressée naguère et me permets de renvoyer à cette étude : voir D. James-Raoul, La parole empêchée dans la littérature arthurienne, Paris, Champion, NBMA, 1997, p. 404-405.

19. Voir, par exemple, Ch. Connochie-Bourgne, "L'ascension de la "pucele vasletee" dans le Roman de Silence d'Heldris de Cornouaille», 2001, p.796-798. Pour tout ce qui concerne l'ambiguïté sexuelle ou linguistique à l'œuvre dans ce roman, les études abondent: voir la bibliographie générale.

20. À titre de comparaison, les capitales colorées les plus employées dans les différents manuscrits d'Érec et Énide sont le E (90 occurrences en moyenne), le Q (88), le L (63), le S (55), le D (50), le A (45), le M (41) et le C (41) : voir R. Middleton, «Coloured Capitals in the Manuscripts of Erec et Enide ", art. cit., p. 169.

21. R. Dragonetti, La Vie de la lettre au Moyen Âge (Le Conte du Graal), Paris, Seuil, 1980, p. 63-83.

22. Voir D. James-Raoul, La parole empêchée dans la littérature arthurienne, op. cit., p. 405.

23. Dans les exemples suivants, je fais figurer en gras les lettrines.

24. Voir le vers 2127.

25. Voir les vers 2083 et 6011, tous deux marqués de surcroît au plan discursif. Au plan logique, seules l'hypothèse (v. 4781) et la concession (v. 3593, 6377) sont par ailleurs représentées.

26. Seules ont été retenues les occurrences où l'adverbe Or, situé dans la diégèse, a un sens véritablement temporel; toutes les occurrences où il soutient de son impulsion le discours (celui du narrateur ou d'un personnage), en particulier en liaison avec un impératif, ont été éliminées. 
27. De même, M. Bolduc constate la tendance exemplaire des miniatures à représenter les dialogues importants entre les différents personnages de ce roman (voir «Images of Romance : The Miniatures of Le Roman de Silence ", art. cit., p. 106-107 et 111).

28. Voir le vers 5879.

29. J'inclus dans ces occurrences la première d'entre elles, qui ouvre le récit au vers 107 , après la fin du prologue.

30. Une seule exception notable est celle présentée par la prolepse qui se termine au vers 3742 .

31. J'emprunte ici la traduction de Fl. Bouchet dans Récits d'amour et de chevalerie (XII ${ }^{e}-\mathrm{XV}^{e}$ siècle), éd. D. Régnier-Bohler, 2000, p. 503.

32. Au sens où l'entend Ph. Hamon, Introduction à l'analyse du descriptif, Paris, Hachette, 1981.

33. Voir Chr. Marchello-Nizia, L'Évolution du français. Ordre des mots, démonstratifs, accent tonique, Paris, Armand Colin, 1995.

34. Ce que R. Middleton appelle la « règle du couplet » me semble souffrir au fil du temps des exceptions de plus en plus nombreuses ("Coloured Capitals in the Manuscripts of Erec et Enide », art. cit., p. 164-166).

35. Si, dans le tableau présenté en Annexe, une seule lettrine est répertoriée à un vers portant un numéro pair, le vers 6582, c'est tout simplement parce que le copiste semble avoir omis un vers dans le groupe de vers précédent, après le vers 6571 ( $d u$ moins, il manque une rime). Et si, inversement, dès le groupe suivant, les lettrines affectent de nouveau des vers dont le numéro est impair, c'est qu'une fois de plus, après le vers 6592, un vers semble avoir été oublié !

36. Bien sûr, la lettrine aurait pu tout aussi bien figurer au vers 3499 , où débute, avec une nouvelle phrase, le discours direct... La part d'arbitraire dans le choix du copiste est ici sensible. 37. D. Boutet, La Chanson de geste, Paris, PUF, 1993, p. 83.

38. Les guillemets fermants en tête des vers énoncés signalent cette fin du discours, sauf s'il s'agit de discours indirect libre, comme au vers 3163.

\section{AUTEUR}

\section{DANIĖLE JAMES-RAOUL}

Université Paris-IV Sorbonne 